\title{
Ubiquitin and SUMO Modifications in Caenorhabditis elegans Stress Response
}

\author{
Krzysztof Drabikowski ${ }^{\star}$
}

Institute of Biochemistry and Biophysics, Polish Academy of Sciences, Warszawa, Poland.

*Correspondence: drabikowski@ibb.waw.pl

https://doi.org/10.21775/9781912530120.21

\begin{abstract}
Post-translational protein modifications by ubiquitin, SUMO and other ubiquitin-like modifiers is common and essential for all eukaryotic organisms. Ubiquitin, SUMO and other ubiquitin-like modifiers are attached to target proteins by a set of related but distinct enzymes including activating enzyme, conjugating enzyme, a ligase and in some cases auxiliary proteins. Both ubiquitin and SUMO proteins regulate most physiological processes in cells and often interdependence of the protein modifications can be observed. Discoveries of ubiquitin and SUMO function have been predominantly driven by studies in cell systems and by in vitro approaches. Investigations of post-translational modifications in Caenorhabditis elegans promises new avenues in ubiquitin and SUMO research. It enables a whole organism approach to study post-translational modifications in development, stress, ageing and in disease models. The biochemical mechanisms of ubiquitin and SUMO modifications are essentially conserved in C. elegans and have been described elsewhere. Thus, this review focuses on emerging research areas where research in C. elegans is advantageous and strongly advances the field of post-translational modifications by ubiquitin and SUMO.
\end{abstract}

\section{Introduction}

The post-translational protein modification by ubiquitin and SUMO is achieved by similar mechanism utilizing a set of homologous but distinct enzymes. (Kerscher et al., 2006; Flotho and Melchior, 2013). Briefly, in the first step, ubiquitin or SUMO proteins are cleaved by the specific carboxyl-terminal hydrolase to produce a carboxyl-terminal diglycine motif. Next, the mature protein is, in an ATP-dependent manner, covalently attached to a cysteine in the activating enzyme E1 via a high-energy thioester bond. The SUMO or ubiquitin moiety is then transferred from E1 to the conjugating enzyme E2, which then attaches the ubiquitin or SUMO to a lysine in the target protein. In case of SUMO, the lysine residue is typically found, but not always, within the consensus sequence $\Psi \mathrm{KXE} / \mathrm{D}$ ( $\Psi$ represents hydrophobic amino acids). SUMO E3 proteins stimulate protein SUMOylation by associating with both, SUMO conjugating enzyme UBC9 and the substrates, to increase the efficiency of the modification reaction. Modification of proteins by ubiquitin is much less specific and any lysine in the target protein can be modified (Mattiroli and Sixma, 2014). Some ubiquitin ligases form thioester intermediate with ubiquitin (HECT) and some serve as a scaffold to bring the E2 close to the modified lysine for 
the conjugation enzyme. The variety of cellular processes regulated by SUMO post-translational modification is as high as by phosphorylation and ubiquitination. Despite this complexity, only few enzymes involved in SUMOylation are known. In comparison, in the ubiquitin conjugation system there are almost thousand proteins involved. Thus, it is expected that the number of proteins involved in the regulation of SUMO modification is much greater. Alternatively, the principle of SUMOylation regulation and specificity is completely different than that of ubiquitination and phosphorylation.

Every dynamic protein modification depends on the developmental stage, cell type and cell cycle stage. Analysis on the level of the entire organism enables a search throughout the entire spectrum of processes and targets involved. Caenorhabditis elegans is an excellent model to decipher the universe of the proteins and processes regulated by SUMO and ubiquitin. C. elegans is a free living soil nematode that in the last 30 years became one of the most important model organisms in developmental biology, neurobiology, genetics and ageing research (Brenner, 2009). It is a higher eukaryote, a metazoan, and all signalling pathways and $60 \%$ of disease related genes present in humans can be found in the worm (Kaletta and Hengartner, 2006). Despite its simplicity and only 959 somatic cells, $C$. elegans forms several different tissues and organs including muscle, intestine, skin, nervous and reproductive systems. C. elegans has a very short life cycle (3.5 days). C. elegans is transparent what enables microscopic analysis. The embryonic development and morphology are mapped with single cell resolution. The nervous system and its connectivity is mapped with single synapse resolution. The genome of $C$. elegans was the first multicellular organism to be sequenced. Several methodologies for C. elegans including mutagenesis and obtaining transgenic animals are well established. RNA interference (RNAi) in C. elegans enables knock-down of protein levels in the entire organism. C. elegans is also the most cost effective metazoan in cultivation allowing whole genome genetic screens and high throughput chemical compound screens. Despite the fact that only very limited number of research groups utilizes C. elegans to study protein modification by ubiquitin and SUMO, research in this model organism brought significant input to the field.

Ubiquitin proteasome system is down-regulated during ageing and in pathological states, for example neurodegenerative diseases. Role of SUMOylation in senescence is less studied but preliminary data point towards mixed effects, both promoting and preventing senescence (Princz and Tavernarakis, 2017). C. elegans is a key model organism in ageing research and advanced knowledge on the basic principles in development of neurodegeneration. Thus, it has the potential to be key model in deciphering and manipulating ubiquitin and SUMO role in ageing.

\section{Components of ubiquitin pathway in Caenorhabditis elegans}

C. elegans ubiquitin system has been described elsewhere (Kipreos, 2005; Papaevgeniou and Chondrogianni, 2014). In this chapter, I will briefly summarize the components. C. elegans genome encodes two loci of ubiquitin genes. Ubq-1 is a polyubiquitin gene with 11 ubiquitin repeats. Repeats are identical except repeat six where threonine at position 9 is substituted by isoleucine. This substitution is not found in other Caenorhabditis species and its significance is unknown. $U b q-2$ is a fusion of $\mathrm{N}$-terminal ubiquitin with $\mathrm{C}$-terminal large ribosomal subunit protein L40. This fusion protein is conserved through Eukaryotes (Finley et al., 1989). In contrast to most genomes the RPL-27 ribosomal protein is fused to a ubiquitin related protein $u b l-1$ (Jones and Candido, 1993). This fusion protein can be also found in protists (Catic et al., 2007). Function of ubl-1 has not been investigated. C. elegans has one ubiquitin activating enzyme UBA1 (Jones et al., 2002; Kulkarni and Smith, 2008). The worm genome encodes 22 proteins homologous of ubiquitin conjugating enzymes and three ubiquitin E2 variants that lack the catalytic site cysteine (Kipreos, 2005). At least 15 of these proteins are responsible for conjugating ubiquitin: UBC-1-3, 6-8 and 12-26 (Jones et al., 2002).

In HECT-domain ligases, ubiquitin moiety is transferred from E2 to E3 conserved cysteine by thioester bond. C. elegans genome encodes nine HECT-domain ligases (hecd-1, hecw-1, oxi-1, herc-1, wwp-1, eel-1 D2085.4, F36A2.13 and Y92H12A.2). These ligases were not studied in detail in worms and the phenotypes are only known from high throughput studies using RNAi. U-box 
domain ubiquitin E3 or E4 ligases are encoded by four genes in worms. $\mathrm{CHN}-1$ is an orthologue of the human CHIP (Khan and Nukina, 2004). CYN-4 is orthologous to human hCyP-60, ufd-2 gene encodes for an $\mathrm{E} 4$ ubiquitin chain assembly factor orthologous to human UBE4B (Hoppe et al., 2004). C. elegans genome encodes for 15 monomeric RING finger proteins but it is not clear if all serve as E3 ubiquitin ligases (Kipreos, 2005). There are six cullin gens cul1-6 (Kipreos, 2005).

None of the SUMO targeted ubiquitin ligases (STUBl) (Prudden et al., 2007; Uzunova et al., 2007) have been identified or studied in the worm but due to the evolutionary conservation of poly SUMO directed poly ubiquitination and further degradation process, one would expect that such ligases exist in C. elegans genome. SUMO chain targeted ubiquitin ligases carry repeats of SUMO interaction motifs (SIMs) (Song et al., 2004, 2005). Repeats of at least three SIMs can be found in over 20 ubiquitin ligases in worms (Drabikowski, unpublished).

In C. elegans two components of the ubiquitin proteasome system in the SCF (Skp1-Cullin-F-box) E3 complex undergone strong gene multiplication. Approximately 520 F-box containing proteins compared to 69 in humans are known (Thomas, 2006; Skaar et al., 2013). SCF complex component Skp1 expanded to 21 members, compared to one in humans. It is proposed that expansion and fast evolution of these proteins enables assembly of a great number of SCF E3 ligases and is a mean to fight intracellular pathogens (Thomas, 2006) (see below).

In C. elegans 45 genes encode de-ubiquitinating enzymes (DUBs), in comparison, in the human genome there is approximately 100 DUBs (Papaevgeniou and Chondrogianni, 2014).

The $C$. elegans genome encodes 14 conserved subunits of the $20 \mathrm{~S}$ core, as well as 18 conserved $19 \mathrm{~S}$ components of the proteasome (Davy et al., 2001). Interference with different proteasomal subunits result in developmental abnormalities (Fraser et al., 2000; Rual et al., 2004; Sönnichsen et al., 2005). Of particular interest is the RPN10 protein, the only proteasome subunit, knock-out of which is not lethal in C. elegans (Shimada et al., 2006) but leads to feminization of the gonad and accumulation of polyubiquitinated proteins (Keith et al., 2016). Decrease in proteasome activity results in activating compensatory mechanisms, which result in net increase of protein quality. In $r p n-10$ mutant C. elegans, the NEDD8 homologue, ned-8, is up-regulated. Depletion of $s k n-1$ in rpn-10 mutants results in developmental arrest due to failure to induce proteasomal subunits expression (Keith et al., 2016). $S k n-1$ is activated on ubiquitin proteasome system UPS down-regulation and promotes expression of proteasomal subunits and aip-1, orthologue of the human arsenite-inducible RNA-associated protein (AIRAP). Proteasome Assembling Chaperones PAC1-4 (Yashiroda et al., 2008) have not been identified in worms. Y57E12AL.6 is an orthologue of human Pomp (proteasome maturation protein, or UMP1). F35G12.12 is an orthologue of human PSMD5 (proteasome 26S subunit, non-ATPase 5) F40G9.17 is an orthologue of human PSMD10 (proteasome 26S subunit, non-ATPase 10) regulatory subunit $p s d m-9$ was up-regulated in worms overexpressing human alpha-synuclein (Vartiainen et al., 2006) suggesting that cellular inclusions induce proteasome assembly and presumably activity.

\section{Components of SUMO pathway in Caenorhabditis elegans}

C. elegans genome encodes one gene, smo- 1 that is essential but due to maternal contribution knockout animals develop into sterile adults (Broday et al., 2004). SMO1 protein is expressed in all cells at all times although the level of expression varies to some extent with the lowest expression in the germline and developing germ cells and the highest in the developing embryo (Drabikowski et al., 2018). Both yeast and worm genomes encode one SUMO gene and the human genome four. Accumulating data suggest that in vertebrates, SUMO1 and SUMO2/3 have different functions. SUMO1 most often forms mono-modification and SUMO $2 / 3$ forms chains and branched chains. Steady state level of SUMO1 modification is high with a little free SUMO1 pool and steady state of SUMO2/3 modification is very low and is attached to targets in stress conditions (Saitoh and Hinchey, 2000). C. elegans SUMO has slightly higher sequence identity with vertebrate SUMO1 than with SUMO $2 / 3,58.8 \%$ and $46.2 \%$, respectively. Evolutionary analysis of SUMO1 protein does not clearly show which homologue it is but the electrostatic 
surface resembles more SUMO2/3 (Surana et al., 2017). Steady state levels of protein modifications by SMO1, both endogenous protein and the SMO1-GFP fusion rescue protein, are very low (Drabikowski et al., 2018). Analysis of SUMO conjugation in worms suggest that SMO1 is involved both in mono- SUMOylation and SUMO chain SUMOylation (Drabikowski et al., 2018). C. elegans SUMO is modifying target proteins in response to stress (see below), which is also characteristic for SUMO2/3 but not SUMO1. C. elegans polySUMO chains were also observed in vitro (Surana et al., 2017). Thus, it is reasonable to assume that C. elegans SUMO performs functions reserved both for SUMO1 and SUMO2/3 in vertebrate systems. Orders of magnitude higher global SUMOylation levels in stress conditions suggest that the stress response is one of the most important functions of SUMO in worms.

The C. elegans SUMO activating enzyme E1 dimer AOS1/UBA2 is encode by aos- 1 and $u b a-2$ genes, respectively. As in other organisms, they are essential genes and depletion by RNAi leads to P0 spindle rotation failures and embryonic lethality (Piano et al., 2002; Sönnichsen et al., 2005). In the surviving animals larval arrest was observed (Piano et al., 2002) as well as several germline development defects (Rual et al., 2004). The sole E2 enzyme UBC9 is encoded by the $u b c-9$ gene (Jones et al., 2002). In C. elegans few SUMO E3 proteins were identified. The PIAS E3 ligase orthologue, Gei-17, was predominantly studied in its involvement in mitosis and cell cycle progression (Pelisch et al., 2014), meiosis (Pelisch et al., 2017; Davis-Roca et al., 2018) and axonal guidance (Chen et al., 2018b). Npp-9 is the orthologue of RanBP2 but has not been studied in C. elegans. ZK1248.11.1 is an orthologue of human NSMCE2 (NSE2/MMS21, SMC5-SMC6 complex SUMO ligase), Pc2, Slx5Slx8 dimer and TOPOROS SUMO ligases have no clear homologue in worms. In C. elegans, four de-SUMOylating enzymes have been identified: ULP-1, ULP-2, ULP-4 and ULP-5. F36D4.5 is an orthologue of human DESI2 (desumoylating isopeptidase 2).

Twenty years of SUMO research revealed only a handful of enzymes involved in SUMO modification, especially the number of SUMO ligases and ligase adaptor proteins is orders of magnitude smaller than those for ubiquitin. On the other hand, SUMO can be conjugated in vitro without E3 involvement. This raises the possibility that in vivo, presence of $\mathrm{E} 3$ is also not required. Psakhey and Jentsch (2012) showed that in case of SUMOylation of DNA repair proteins, all proteins in the vicinity are SUMOylated suggesting that regulation of modification is spatiotemporal and not relaying on a big group of proteins providing substrate specificity (Psakhye and Jentsch, 2012). Several lines of evidence show that SUMOylation is dramatically increased in stress conditions (see below) raising the possibility that also in stress, specificity of SUMOylation comes from a specific trigger in a specific local environment and not from distinct set of proteins as in case of ubiquitination and phosphorylation.

\section{The universe of proteins modified by ubiquitin and SUMO}

Proteomics analysis of the entire organism in all its developmental stages enables deciphering the set of proteins involved in an analysed process. In C. elegans most SUMOylation takes place in the nucleus but the number cytoplasmic proteins modified exceeds the number of nuclear ones. Cytosolic SUMO targets include many metabolic enzymes, cytoskeleton components and big protein complexes of ribosome and proteasome. A big group of SUMO targets are mitochondrial and secreted proteins and Drabikowski et al. (2018) suggests that these targets could undergo SUMO directed polyubiquitination and degradation. Independent analyses by Hendriks et al. (2014) and Drabikowski et al. (2018) showed very high evolutionary conservation of SUMOylation targets among species. Thus, Drabikowski et al. (2018) suggested a novel approach to predict potential SUMOylation of a protein based on experimental proof of SUMOylation of this protein in other species. This prediction estimated the number of SUMOylated proteins to be at least $15-20 \%$ of the proteome. The dbPMT database currently lists approximately 100,000 unique ubiquitination sites in human in 10,000 proteins (Huang et al., 2016). This represents approximately $15 \%$ of the proteome, comparable number to the number of SUMOylated proteins estimated by Drabikowski et al. (2018). Ubiquitin targets in C. elegans have not been analysed on a global scale and such an analysis would greatly 
contribute to deciphering the universe of ubiquitin targets in other organisms.

\section{SUMO and ubiquitin in stress response and ROS signalling}

SUMO and ubiquitin activating and conjugating enzymes as well as some E3 ligases rely on the catalytic cysteine that forms a thioester bond with the C-terminus of SUMO and ubiquitin proteins. Ubiquitin- and SUMO-specific proteases have also catalytic cysteine. Cysteines are prone to oxidation and thus allowing reactive oxygen species to, at low physiological conditions, regulate conjugation and de-conjugation processes. Indeed, Bossis and Melchior (2006) showed that ROS reversibly blocks SUMO conjugation by forming a disulfide bond between E1 and E2. Stankovic-Valentin et al. (2016) showed that SUMO E1-E2 oxidation is an essential redox switch in oxidative stress. The SUMO E2 UBC9 with D100A mutation near the catalytic site remains catalytically active but is unable to form a disulfide bond with E1. Cells with UBC9 replaced with the mutant have compromised survival under oxidative stress (Stankovic-Valentin et al., 2016). A disulfide bond between the ubiquitin E2 Cdc34 and Aos1 has also been described (Doris et al., 2012). These results suggest that redox regulation of protein-protein modifications by ubiquitin and ubiquitin like molecules is a crucial factor in regulating these modifications and in turn, they are essential components of ROS signalling.

C. elegans as a free-living soil nematode had to adapt to various external and internal stress stimuli. One of the pillars of cellular homeostasis is maintaining protein homeostasis, the proteostasis. Proteostasis depends on the regulation and balance between proteins synthesis and degradation achieved by the ubiquitin proteasome system and autophagy.

Mounting evidence suggests that SUMOylation is greatly increased in stress conditions. Golebiowski et al. (2009) showed global changes in SUMOylation on heat stress in yeast. In plants heat shock also induces SUMOylation (Miller et al., 2013) along with drought (Catala et al., 2007) and osmotic stress (Castro et al., 2016).

In C. elegans the increase in SUMOylated species on heat shock is one of the highest in stress conditions. (Drabikowski et al., 2018). Heat shock induces massive deactivation of de-SUMOylating enzymes (Pinto et al., 2012). This phenomenon cannot explain increased SUMOylation level on stress. All other stressors, even those activating heat shock response, are not expected to deactivate the de-SUMOylating enzymes and thus the mechanism of increased SUMOylation on heat shock does not primarily depend on deactivation of de-SUMOylating enzymes. Troponin like calcium binding protein PAT-10 is essential for thermotolerance of worms. Transcription of pat-10 is directly orchestrated by heat shock factor HSF-1 and PAT-10 provides stability to the cytoskeleton in heat shock. Depletion of PAT-10 results in collapse of actin cytoskeleton (Baird et al., 2014). PAT-10 is SUMOylated in worms (Drabikowski et al., 2018) and one can speculate that the role of SUMOylation is in actin filaments assembly/disassembly (see below).

Reactive oxygen species emerge as key modulator of the ubiquitin and SUMO modification pathways. Reactive oxygen species induce reversible $26 \mathrm{~S}$ proteasome disassembly leading to attenuation of ubiquitin mediated proteolysis (Livnat-Levanon et al., 2014). Proteasomal disfunction activates antioxidative stress response enzymes GST-4 and SOD expression. Reactive oxygen species response is orchestrated by the transcription factor SKN-1, which regulates the transcription of phase II detoxification enzymes. SKN-1 interacts with MDT-15 to facilitate oxidative metabolism and promote lifespan. MDT-15 is a component of the Mediator complex, a coactivator involved in regulated gene transcription of nearly all RNA polymerase II-dependent genes. In parallel to SKN-1, MDT-15 interacts with NHR-49 to promote ROS response and expression of gst-4. Both NHR-49 and MDT-15 are SUMOylated in C. elegans (Drabikowski et al., 2018). SKN-1 has not been found to be SUMOylated but it contains a canonical SUMO interacting motif (SIM) ILVISS at position 57. One can predict that SUMOylation of NHR-49 and MDT-15 brings together SKN-1 with these proteins. Direct interaction of SKN-1 and NHR- 49 has not been observed (Hu et al., 2018) but interaction through SUMO modified NHR-49 and MDT-15 would explain the interaction and as well provide an oxidation dependent regulation of this interaction. It is not known if ROS induces NHR-49 SUMOylation or block it and thus activating it. NHR-49 is 
also a key regulator of fatty acid metabolism in basal and starvation states (Van Gilst et al., 2005; Ratnappan et al., 2014). NHR-49, in concert with EGL-4 is a regulator of lysosomal lipid storage during short term fasting (Huang et al., 2014). Thus, NHR-49 provides a link between SUMO and regulation of metabolism and starvation stress response.

Arsenite increases the amount of polyubiquitinated species in vertebrate cells (Kirkpatrick et al., 2003) and in C. elegans (Stanhill et al., 2006). The mechanisms of arsenite toxicity are very complex and not fully understood. It can replace phosphate in several reactions, it can react with thiols in proteins and inhibit their activity and it can generate ROS. Accumulation of polyubiquitinated species appears to happen within hours of exposure and polysumoylation within minutes (Drabikowski et al., 2018). Arsenite is also the fastest and one of the strongest SUMOylation inducers in C. elegans (Drabikowski et al., 2018). Thus, one can speculate that SUMOylation precedes ubiquitination in response to arsenite toxicity. Paraquat induces SUMOylation to a much smaller extent than sodium arsenite suggesting that oxidative damage induced by sodium arsenite is only a small component of the toxicity of this compound. Both sodium arsenite and paraquat induce expression of phase II detoxification enzymes (Oliveira et al., 2009).

Arsenite inducible protein (AIP) associated with 19S regulatory particle (Stanhill et al., 2006), AIP-1 and it's human homologue AIRAP regulates the proteasome to counteract proteotoxicity induced by arsenite. AIRAP containing proteasomes are more active. In C. elegans, aip-1 ameliorates amyloidbeta peptide toxicity (Hassan et al., 2009). Aip-1 expression is under control of $s k n-1$ (Ferguson et al., 2010).

Prolonged, high ER stress $(30 \mu \mathrm{M}$ tunicamycin, 5 hours) reduces the level of SUMOylation (Lim et al., 2014). In contrast, in the recovery phase of shorter and weaker ER stress $(5 \mu \mathrm{M}$ tunicamycin, 0.5 hours) SUMOylation is increased, too much smaller extent than heat shock or arsenite treatment, though (Drabikowski et al., 2018). In C. elegans, as in other organisms, calreticulin (ctr-1) is up-regulated on ER stress. Lim et al. (2014) proposed a model were SUMO modifies the stress inducible transcription factor, XBP-1, and thus repressing its activity. On ER stress, SUMOylation of XBP-1 is reduced and the transcription factor is activated.

Drabikowski et al. (2018) showed that all tested stressors including heat shock, UV, arsenite, ROS, ER stress and osmotic stress, induce SUMOylation to various extent (Drabikowski et al., 2018) In yeast, the dramatic increase in levels of SUMOylation on heat stress is dependent on the SUMO ligase Siz1 (Lewicki et al., 2015). The C. elegans orthologue of Siz1, GEI-17 is involved in SUMO dependent complex assembly during mitosis, meiosis and DNA repair but it's role in SUMO stress response has not been addressed. All stress response pathways are involved in maintaining protein homeostasis and ageing.

\section{Protein homeostasis and ageing}

Ageing is associated with, and at least partially caused by decreased capability of an organism to maintain protein homeostasis. C. elegans is one of the key model organisms in ageing research. Several ageing related signalling pathway have been deciphered in worms. In worms ageing causes decreased response to multiple stresses and fail to activate stress responses (Dues et al., 2016).

Dietary restriction increase lifespan in various organisms tested. In C. elegans, the WWP-1 HECT-domain ubiquitin ligase is necessary for life extension of dietary restricted worms (Carrano et al., 2009). Reduction of WWP-1 abolishes dietary restriction extension of lifespan and overexpression of WWP-1 leads to elongation of lifespan in non-restricted animals. This extension depends on E3 ubiquitin ligase activity and interaction with UBC-18, an E2 ubiquitin conjugating enzyme. UBC-18 is SUMOylated in worms (Drabikowski et al., 2018) and WWP-1 caries two sumo interacting motifs (Drabikowski, unpublished) raising the possibility that the interaction of WWP-1 with UBC-9 is SUMO regulated. The downstream target for WWP-1 is KPL-1, a Krüppel like transcription factor (Carrano et al., 2014). Another ubiquitin E3 ligase linking protein homeostasis and ageing is the quality control E3, CHIP. Insulin receptor DAF-2 is a direct target of CHIP were monoubiquitination of the insulin-like receptor results in its internalization and lysosomal degradation. In aged animals, CHIP is recruited to misfolded proteins resulting in increased insulin signalling and accelerated ageing 
(Tawo et al., 2017). Downstream of insulin/IGF signalling (IIS) pathway is the FOXO transcription factor DAF-16 that regulates expression of stress resistance genes and are essential mediators of longevity. IIS down-regulates FOXO transcription factors. MDT-15 also interacts with SKN-1 to facilitate oxidative metabolism and promote lifespan in an nhr-49 independent manner (Goh et al. 2014; Pang et al. 2014). NHR-49 and MDT-15 enhance expression of SKN-1. The C. elegans de-ubiquitinating enzyme MATH-33 deubiquitinates DAF-16, the worm FOXO, counteracting polyubiquitination by RLE-1 E3 ligase and its subsequent proteasomal degradation. Stabilization of DAF-16 enables expression of pro longevity genes (Heimbucher et al., 2015). Similarly, RLE-1 mutations prolong lifespan ( $\mathrm{Li}$ et al., 2007). In adult worms starvation induces adult reproductive diapause that results in delaying the reproductive onset and extends the lifespan three fold. In turn, NHR-49 expression is up-regulated by DAF-16 and TCER-1 in germline-ablated animals but also regulates subcellular localization of KRI-1 that is a regulator of expression of DAF-16 and TCER-1 (Ratnappan et al., 2014). Furthermore, SKN-1 and ELT-2 are regulated by the BRAP2 ubiquitin ligase in the expression of phase II detoxification enzymes (Hu et al., 2018). BRAP2 also regulates F-box protein XREP-4. XREP-4 interacts with the ubiquitin ligase component SKR-1 and the SKN-1 principal repressor WDR-23. WDR-23 functions as part of a CUL-4/DDB-1 ubiquitin ligase complex that negatively regulates accumulation and hence, transcriptional activation activity of the SKN-1. Loss of xrep-4 inhibits the $s k n$-1-dependent expression of detoxification genes in response to prooxidants and decreases survival of oxidative stress (Wu et al., 2017).

Reproductive diapause results in apoptotic death of most of the germline with the exception of a small population of germ cells that can regrow the germline after feeding resumes. Entry and exit from the adult reproductive diapause require the NHR-49 transcription factor (Angelo and Van Gilst, 2009). Since NHR-49 is SUMOylated, it raises the possibility of regulation of starvation response by the SUMO pathway. Insulin/IGF signalling pathway has also negative impact on lifespan independent of DAF-16 by promoting SUMOylation of the germline protein CAR-1 (Cytokinesis/
Apoptosis/RNA-binding protein 1) and thereby accelerating ageing (Moll et al., 2018).

Independent of IS extension of lifespan was observed in mutants von Hippel-Lindau tumour suppressor homologue. VHL-1 is a cullin E3 ubiquitin ligase that negatively regulates the hypoxic response by promoting ubiquitination and degradation of the hypoxic response transcription factor HIF-1. Loss of VHL-1 significantly increased lifespan and enhanced resistance to polyglutamine and amyloid beta toxicity (Mehta et al., 2009). Depletion of another ubiquitin ligase, MEL-26, leads to extension of the lifespan (Chen et al., 2018a). MEL-26 is an orthologue of KLHK22 ubiquitin ligase and activates the mechanistic target of rapamycin complex 1 (mTORC1). The parkin E3 ligase, PDR-1, is required for mitophagy dependent increase in longevity (Palikaras et al., 2015).

In a large proteomics study addressing changes in the proteome on ageing in C. elegans, the $19 \mathrm{~S}$ and $20 \mathrm{~S}$ proteasome was elevated in older animals along with many E3 ligases and other components of ubiquitin-proteasome system (Walther et al., 2015). Activation of $20 \mathrm{~S}$ proteasome leads to extension of lifespan and resistance to stress. Proteasome activation delays ageing in vitro and in vivo (Chondrogianni et al., 2014). Vilchez et al. (2012) analysed the proteasomal activity in $g l p-1$ long living mutants lacking germline and under dietary restriction conditions. All types of protease activity of the proteasome were higher in the $g l p-1$ mutants. The only proteasomal subunit which expression was significantly higher was the RPN-6.1 regulatory subunit of the 19S proteasome. RPN-6.1 stabilizes the otherwise loose interaction between $20 \mathrm{~S}$ and 19S proteasome cap. Transcription of RPN-6.1 is mediated by DAF-16. Knock down of RPN-6.1 in wild type worms decreased lifespan and overexpression led to increased resistance to oxidative stress or heat shock and to extension of the lifespan (Vilchez et al., 2012). In C. elegans, RPN-6.1 is a target of SUMO (Drabikowski et al., 2018) suggesting that SUMO might have a direct role regulating proteasomal activity by stabilizing or de-stabilizing interactions between 20 S and 19S proteasomal subunits.

Much less in known about involvement of SUMO in proteostasis and ageing in worms but one would expect its role is as important and widespread as that of ubiquitin. First, it is highly 
probable that SUMOylation regulates $26 \mathrm{~S}$ proteasomal assembly/disassembly as well as involved in regulation of key transcription factors involved in expression of ubiquitin proteasomal system genes and stress response genes. Furthermore, at least $20 \%$ of SUMO targets in C. elegans identified by Drabikowski et al. (2018) is localized to the cellular compartments where there is no SUMOylation and the explanation for such outcome is that these substrates undergo SUMO directed poly ubiquitination and subsequent proteasomal degradation as a quality control mechanism. Accumulation of SUMO substrates in human cells on proteasome inhibition (Hendriks et al., 2014) favours this interpretation. SUMO modification of the mevalonate pathway enzyme HMGS-1 regulates metabolism during C. elegans ageing (Sapir et al., 2014). The mevalonate pathway is one of the key cascades in metabolism and HMGS-1 mediates the first step in the mevalonate pathway. In worms, HMGS-1 is SUMOylated in an age dependent manner. Early in life, activity of the ULP-4 de-SUMOylating enzyme keeps HMGS-1 in a un-SUMOylated state and presumably active. Later, mitochondrial sequestration of ULP-4 leads to increased SUMOylation of HMGS-1 and its inactivation (Sapir et al., 2014). Furthermore, HMGS-1 levels are regulated by the ubiquitin-proteasome system. Drabikowski et al. (2018) identified 159 C. elegans SUMO targets with the GO term 'metabolism. Thus, it is highly probable that direct regulation of metabolism by SUMO and not just by regulating transcription factors involved in metabolic enzymes expression, is much more common than previously anticipated. For example, several ROS response proteins, glutathione synthetase GSS-1, glutathione S-transferases GST-1,6,7 36 have been identified as direct SUMO targets (Drabikowski et al., 2018).

\section{Infections and innate immunity}

It is well established in many biological systems that bacterial and viral pathogens target ubiquitin, SUMO and NEDD8 pathway to evade response and reprogram cell metabolism for own purpose (Ribet and Cossart, 2018). Bacteria do not have eukaryotic type ubiquitin and ubiquitin-like systems but evolved to interfere with the host systems. Several bacterial toxins down-regulate ubiquitination by targeting E1, E2 and E3 enzymes. On the other hand, worms are an infection model for several human bacterial pathogens and are increasingly utilized in deciphering of bacterial pathogenicity (Irazoqui et al., 2010a) and for antimicrobial compound screens (Squiban and Kurz, 2011). Since nematodes are devoid of specialized immune cells, they lost the NFKB pathway (Irazoqui et al., 2010b). C. elegans enables the study of innate immunity and dissecting ubiquitination functions in host defence independent of NFKB pathway.

Ubiquitin involvement in C. elegans innate immunity was proposed by Thomas (2006) concluding that great expansion of genes coding for F-box proteins and SCX proteins could be a host defence mechanism against pathogens. Indeed, in transcriptomics analysis of worms infected with bacterial pathogens colonizing the $C$. elegans gut, S. marcescens, E. faecalis and P. luminescens, several F-box proteins as well as other components of the ubiquitin conjugating machinery were up-regulated on infection (Engelmann et al., 2011). Genes up-regulated in Orsay virus and microsporidia Nematocida parisii are enriched by the F-box proteins (Bakowski et al., 2014). In a whole genome RNAi screen for genes involved in innate immunity response to the infection by a pathogenic fungus Drechmeria coniospora, several genes from the ubiquitin proteasome pathway were found, including let-70, hecd-1, prp-19, rbpl-1 ubiquitin ligases, dcaf-1, skr-1 ubiquitin ligase complex components, proteasome components pas-3, pas-5, pbs-2, and ubiquitin specific protease usp-39 (Zugasti et al., 2016). Intracellular pathogens are expected to cause direct and severe damage to proteostasis. Nematocidal parisii in a species of microsporidia fungi that is an intracellular pathogen specifically infecting Caenorhabditis nematodes (Félix et al., 2011). N. parisii infection induces expression of several ubiquitin ligase components cul-6, skr-3, skr-4, and skr-5. In a forward genetics screen Reddy et al. identified pals-22 mutant. Pals-22 is a repressor of cul-6 gene. Increased stress resistance is dependent on CUL6 and independent of heat shock response pathway and insulin pathway (Reddy et al., 2017). Ubiquitin modifications provide defence against the intracellular microsporidia pathogen Nematocida parisii by the proteasome system and by autophagy (Bakowski et al., 2014). Some of N. 
parisii cells are decorated by ubiquitin and this is dependent on SCF ubiquitin ligase components cul-6, skr-3 and skr-5. Most parasites are not decorated and in the infected worms treated with a microsporidial drug, suggesting a mechanism employed by the fungus to evade recognition and ubiquitination. Bacterial strategies to evade or overtake ubiquitin or SUMO systems have not been studied in C. elegans but worms would be an interesting model to study such interactions. For example, Salmonella enterica and Shigella flexneri dampens UBC9 activity and on the other hand infect C. elegans (Haskins et al., 2008; George et al., 2014). S. enterica infection results in substantial increase in reactive oxygen species in the worm gut that can be reversed by application of antioxidants (Sem and Rhen, 2012). It is tempting to speculate that the ubiquitin proteasome pathway and SUMO pathway are the molecular targets of Salmonella induced ROS. Pathogenicity of Salmonella enterica results in germ line cells apoptosis, unfolded protein response (UPR) and blocking of UPR leads to fast killing of worms by $S$. enterica. UPR results in ubiquitination of misfolded proteins and subsequent degradation by the proteasome.

Nematodes have also their own viral pathogens: the Orsay, Santeuil and Le Blanc viruses (Félix et al., 2011; Franz et al., 2014). C. elegans has been also used as an infection model for the vesicular stomatitis virus (VSV) and Flock house virus (FHV). RNAi pathway provides one line of defence against RNA viruses but not the only one. Ubiquitin modifications provide defence against Orsay virus infection (Bakowski et al., 2014). Infection of Orsay virus results in up-regulation of several ubiquitin ligase adaptor proteins as well as SCF components cul-6, skr-4, skr-5 (Chen et al., 2017), RNAi knock down of SCF components increase the virus load. On the other hand, disrupting the entire ubiquitin proteasome system by RNAi reduced virus load, suggesting that the Orsay virus requires UPS for its replication. Viruses target the SUMOylation pathway by disrupting the PML nuclear bodies. In C. elegans genome, the PML protein has not been identified but SUMO rich nuclear bodies have been observed (Drabikowski et al., 2018). Effect of C. elegans specific viruses on the SUMO pathway has not been studied.

\section{SUMO in protein complex assembly}

Involvement of SUMO in protein complex formation was first shown for the PML bodies where the PML protein is both SUMOylated and contains SUMO interacting motifs (Shen et al., 2006). Mounting data suggests that this role of SUMO is much more common and important for cellular physiology providing a molecular glue combining proteins to form a complex or blocking interactions of proteins that otherwise would form one. It was first shown by Psakhye and Jentsch (2012) that, in yeast, in response to double-stranded DNA break, proteins in the repair pathway undergo a wave of SUMOylation and the entire set of proteins forming the repair complex is modified. These modifications at multiple sites are required for double-stranded break complex assembly.

In C. elegans, RNAi enables to knock down genes involved in germline development and thus the study of meiosis. During C. elegans oocyte meiosis, a multiple protein ring complex (RC) is assembled between to homologous chromosomes. The ring complex is required for chromosome cohesion through the action of chromokinesin KLP-19. KLP-19 is SUMOylated by the GEI-17/PIAS SUMO E3 ligase and GEI-17 is required for KLP-19 recruitment to the ring complex. GEI-17 along another component of RC, the kinase BUB-1 carry SUMO interacting motifs. Auto- SUMOylated GEI-17 and KLP-19 interact with SIMs from BUB-1 and GEI-17 and form the complex (Pelisch et al., 2017).

The dosage compensation process ensures that in C. elegans hermaphrodites, with two $\mathrm{X}$ chromosomes, there is the same amount of proteins expressed from genes encoded on the $\mathrm{X}$ chromosomes as in males which have only one $\mathrm{X}$ chromosome. This is achieved by the dosage compensation complex DCC of 10 proteins which is partially homologous to condensin. The DCC binds to both $\mathrm{X}$ chromosomes in hermaphrodites, reducing recruitment of RNA polymerase II and thus reducing the transcription by half. Multiple subunits of the dosage compensation complex are SUMOylated and most, except two, have multiple SUMO interacting motifs. The recruiting factor SDC-2 does not require SUMOylation to bind to the $\mathrm{X}$ chromosome but subsequent steps do and depletion of SUMO results in diffused nuclear 
localization of these proteins and not to specific foci (Pferdehirt and Meyer, 2013).

Besides providing protein glue facilitating protein-protein interactions, SUMO modification can also prevent or weaken interactions. For example, in intermediate filament assembly, SUMOylation of IFB-1 maintains the soluble pool of the IFA-1/ IFB-1 heterodimer and de-SUMOylation results in intermediate filament polymerization (Kaminsky et al., 2009). In support, knock down of UBC-9 by RNAi results in short thick filaments (Lim et al., 2014). In C. elegans, the adherens junctions are dynamic membrane bound structures providing both flexibility and rigidity of cell-cell contacts in embryogenesis and adult tissue remodelling. The main component of adherens junctions, the E-cadherin HMG-1, is SUMOylated in C. elegans (Tsur et al., 2015). Thus, assembly and remodelling of adherens junctions relies on a cycle of SUMOylation and desumoylation of E-cadherin.

In worms and in other organisms, modification by SUMO were shown to switch ON/OFF protein-protein interactions required for assembly of several protein complexes. These discoveries might still just be the tip of an iceberg. Thousands of proteins have been shown to be SUMOylated and Drabikowski et al. (2018) predicted that at least $15-20 \%$ of eukaryotic proteomes are modified by SUMO. In C. elegans, the SUMO interaction motifs, $[\mathrm{VI}] \times[\mathrm{VI}][\mathrm{VI}]$ or $[\mathrm{VI}][\mathrm{VI}] \times[\mathrm{VI}]$, can be found in over 12,000 proteins (Drabikowski, unpublished) which roughly represents half of the proteome. This number is comparable to the number of SIMs identified in the yeast proteome. Thus, the potential number of ON/OFF switchable interaction between SUMO modified proteins and proteins carrying SUMO interacting motif is enormous and largely unexplored.

\section{Conclusions}

Forty years of research in ubiquitin and 20 years in SUMO proved their critical role in maintaining cellular proteostasis and thereby regulating virtually all aspects of physiology and pathology. Research in Caenorhabditis elegans enables a whole organism approach to study an entire universe of posttranslational modifications by ubiquitin-like modifiers in all developmental stages and in all tissues that otherwise would be missed in cell culture experiments.
Whole organism approach is particularly useful in studying physiological processes in the germline and embryonic development, behaviour as well as organismal stress response and ageing. Research in worms showed crucial role of ubiquitin and SUMO modification systems in maintaining proteostasis in healthspan and lifespan of an organism. SUMOylation and ubiquitination processes interact and mutually regulate each other as well as interacting with stress response and metabolic pathways. Discoveries of ubiquitin and SUMO modifications as well as their functions can be extrapolated to other organisms showing relevance of studying them in C. elegans. In future, C. elegans might be particularly useful in high throughput compound screens for molecules affecting ubiquitin-proteasome system and SUMO system that will result in new drugs for: proteasome inhibitors for cancer treatment, compounds increasing proteostasis capacity for extending healthspan and compounds boosting innate immunity in bacterial and viral infections. Emerging picture from C. elegans and other research is that SUMO functions in two areas of cell physiology: providing switchable molecular glue/repellent for complex assembly and regulating proteostasis in concert with ubiquitin.

\section{References}

Angelo, G., and Van Gilst, M.R. (2009). Starvation protects germline stem cells and extends reproductive longevity in C. elegans. Science 326, 954-958. https://doi. org/10.1126/science.1178343.

Baird, N.A., Douglas, P.M., Simic, M.S., Grant, A.R., Moresco, J.J., Wolff, S.C., Yates, J.R., Manning, G., and Dillin, A. (2014). HSF-1-mediated cytoskeletal integrity determines thermotolerance and life span. Science 346, 360-363. https://doi.org/10.1126/science.1253168.

Bakowski, M.A., Desjardins, C.A., Smelkinson, M.G., Dunbar, T.L., Dunbar, T.A., Lopez-Moyado, I.F., Rifkin, S.A., Cuomo, C.A., and Troemel, E.R. (2014). Ubiquitin-mediated response to microsporidia and virus infection in C. elegans. PLOS Pathog. 10, e1004200. https://doi.org/10.1371/journal.ppat.1004200.

Bossis, G., and Melchior, F. (2006). Regulation of SUMOylation by reversible oxidation of SUMO conjugating enzymes. Mol. Cell 21, 349-357.

Brenner, S. (2009). In the beginning was the worm. Genetics 182, 413-415. https://doi.org/10.1534/ genetics.109.104976.

Broday, L., Kolotuev, I., Didier, C., Bhoumik, A., Gupta, B.P., Sternberg, P.W., Podbilewicz, B., and Ronai, Z. (2004). The small ubiquitin-like modifier (SUMO) is required for gonadal and uterine-vulval morphogenesis in Caenorhabditis elegans. Genes Dev. 18, 2380-2391.

Carrano, A.C., Liu, Z., Dillin, A., and Hunter, T. (2009). A conserved ubiquitination pathway determines longevity 
in response to diet restriction. Nature 460, 396-399. https://doi.org/10.1038/nature08130.

Carrano, A.C., Dillin, A., and Hunter, T. (2014). A Krüppellike factor downstream of the E3 ligase WWP-1 mediates dietary-restriction-induced longevity in Caenorhabditis elegans. Nat. Commun. 5, 3772. https://doi. org/10.1038/ncomms4772.

Castro, P.H., Couto, D., Freitas, S., Verde, N., Macho, A.P., Huguet, S., Botella, M.A., Ruiz-Albert, J., Tavares, R.M., Bejarano, E.R., et al. (2016). SUMO proteases ULP1c and ULP1d are required for development and osmotic stress responses in Arabidopsis thaliana. Plant Mol. Biol. 92, 143-159. https://doi.org/10.1007/s11103-0160500-9.

Catala, R., Ouyang, J., Abreu, I.A., Hu, Y., Seo, H., Zhang, X., and Chua, N.H. (2007). The Arabidopsis E3 SUMO ligase SIZ1 regulates plant growth and drought responses. Plant Cell 19, 2952-2966.

Catic, A., Sun, Z.Y., Ratner, D.M., Misaghi, S., Spooner, E., Samuelson, J., Wagner, G., and Ploegh, H.L. (2007). Sequence and structure evolved separately in a ribosomal ubiquitin variant. EMBO J. 26, 3474-3483.

Chen, J., Ou, Y., Yang, Y., Li, W., Xu, Y., Xie, Y., and Liu, Y. (2018a). KLHL22 activates amino-acid-dependent mTORC1 signalling to promote tumorigenesis and ageing. Nature 557, 585-589. https://doi.org/10.1038/ s41586-018-0128-9.

Chen, K., Franz, C.J., Jiang, H., Jiang, Y., and Wang, D. (2017). An evolutionarily conserved transcriptional response to viral infection in Caenorhabditis nematodes. BMC Genomics 18, 303. https://doi.org/10.1186/ s12864-017-3689-3.

Chen, S.Y., Ho, C.T., Liu, W.W., Lucanic, M., Shih, H.M., Huang, P.H., and Cheng, H.J. (2018b). Regulation of axon repulsion by MAX-1 SUMOylation and AP-3. Proc. Natl. Acad. Sci. U.S.A. 115, E8236-E8245. https://doi.org/10.1073/pnas.1804373115.

Chondrogianni, N., Sakellari, M., Lefaki, M., Papaevgeniou, N., and Gonos, E.S. (2014). Proteasome activation delays aging in vitro and in vivo. Free Radic. Biol. Med. 71, 303-320. https://doi.org/10.1016/j. freeradbiomed.2014.03.031.

Davis-Roca, A.C., Divekar, N.S., Ng, R.K., and Wignall, S.M. (2018). Dynamic SUMO remodeling drives a series of critical events during the meiotic divisions in Caenorhabditis elegans. PLOS Genet. 14, e1007626. https://doi.org/10.1371/journal.pgen.1007626.

Davy, A., Bello, P., Thierry-Mieg, N., Vaglio, P., Hitti, J., Doucette-Stamm, L., Thierry-Mieg, D., Reboul, J., Boulton, S., Walhout, A.J., et al. (2001). A proteinprotein interaction map of the Caenorhabditis elegans 26S proteasome. EMBO Rep. 2, 821-828. https://doi. org/10.1093/embo-reports/kve184.

Doris, K.S., Rumsby, E.L., and Morgan, B.A. (2012). Oxidative stress responses involve oxidation of a conserved ubiquitin pathway enzyme. Mol. Cell. Biol. 32, 4472-4481. https: //doi.org/10.1128/MCB.0055912.

Drabikowski, K., Ferralli, J., Kistowski, M., Oledzki, J., Dadlez, M., and Chiquet-Ehrismann, R. (2018). Comprehensive list of SUMO targets in Caenorhabditis elegans and its implication for evolutionary conservation of SUMO signaling. Sci. Rep. 8, 1139. https://doi. org/10.1038/s41598-018-19424-9.

Dues, D.J., Andrews, E.K., Schaar, C.E., Bergsma, A.L., Senchuk, M.M., and Van Raamsdonk, J.M. (2016). Aging causes decreased resistance to multiple stresses and a failure to activate specific stress response pathways. Aging 8, 777-795. https://doi.org/10.18632/ aging.100939.

Engelmann, I., Griffon, A., Tichit, L., Montañana-Sanchis, F., Wang, G., Reinke, V., Waterston, R.H., Hillier, L.W., and Ewbank, J.J. (2011). A comprehensive analysis of gene expression changes provoked by bacterial and fungal infection in C. elegans. PLOS ONE 6, e19055. https://doi.org/10.1371/journal.pone.0019055.

Félix, M.A., Ashe, A., Piffaretti, J., Wu, G., Nuez, I., Bélicard, T., Jiang, Y., Zhao, G., Franz, C.J., Goldstein, L.D., et al. (2011). Natural and experimental infection of Caenorhabditis nematodes by novel viruses related to nodaviruses. PLOS Biol. 9, e1000586. https://doi. org/10.1371/journal.pbio.1000586.

Ferguson, A.A., Springer, M.G., and Fisher, A.L. (2010). skn-1-Dependent and -independent regulation of aip-1 expression following metabolic stress in Caenorhabditis elegans. Mol. Cell. Biol. 30, 2651-2667. https://doi. org/10.1128/MCB.01340-09.

Finley, D., Bartel, B., and Varshavsky, A. (1989). The tails of ubiquitin precursors are ribosomal proteins whose fusion to ubiquitin facilitates ribosome biogenesis. Nature 338, 394-401. https://doi.org/10.1038/338394a0.

Flotho, A., and Melchior, F. (2013). Sumoylation: a regulatory protein modification in health and disease. Annu. Rev. Biochem. 82, 357-385. https://doi. org/10.1146/annurev-biochem-061909-093311.

Franz, C.J., Renshaw, H., Frezal, L., Jiang, Y., Félix, M.A., and Wang, D. (2014). Orsay, Santeuil and Le Blanc viruses primarily infect intestinal cells in Caenorhabditis nematodes. Virology 448, 255-264. https://doi. org/10.1016/j.virol.2013.09.024.

Fraser, A.G., Kamath, R.S., Zipperlen, P., MartinezCampos, M., Sohrmann, M., and Ahringer, J. (2000). Functional genomic analysis of C. elegans chromosome I by systematic RNA interference. Nature 408, 325-330. https://doi.org/10.1038/35042517.

George, D.T., Behm, C.A., Hall, D.H., Mathesius, U., Rug, M., Nguyen, K.C., and Verma, N.K. (2014). Shigella flexneri infection in Caenorhabditis elegans: cytopathological examination and identification of host responses. PLOS ONE 9, e106085. https://doi.org/10.1371/journal. pone. 0106085 .

Golebiowski, F., Matic, I., Tatham, M.H., Cole, C., Yin, Y., Nakamura, A., Cox, J., Barton, G.J., Mann, M., and Hay, R.T. (2009). System-wide changes to SUMO modifications in response to heat shock. Sci. Signal. 2, ra24. https://doi.org/10.1126/scisignal.2000282.

Haskins, K.A., Russell, J.F., Gaddis, N., Dressman, H.K., and Aballay, A. (2008). Unfolded protein response genes regulated by CED-1 are required for Caenorhabditis elegans innate immunity. Dev. Cell 15, 87-97. https:// doi.org/10.1016/j.devcel.2008.05.006.

Hassan, W.M., Merin, D.A., Fonte, V., and Link, C.D. (2009). AIP-1 ameliorates beta-amyloid peptide toxicity in a Caenorhabditis elegans Alzheimer's disease model. Hum. Mol. Genet. 18, 2739-2747. 
Heimbucher, T., Liu, Z., Bossard, C., McCloskey, R., Carrano, A.C., Riedel, C.G., Tanasa, B., Klammt, C., Fonslow, B.R., Riera, C.E., et al. (2015). The deubiquitylase MATH-33 controls DAF-16 stability and function in metabolism and longevity. Cell Metab. 22, 151-163. https://doi.org/10.1016/j.cmet.2015.06.002.

Hendriks, I.A., D’Souza, R.C., Yang, B., Verlaan-de Vries, M., Mann, M., and Vertegaal, A.C. (2014). Uncovering global SUMOylation signaling networks in a sitespecific manner. Nat. Struct. Mol. Biol. 21, 927-936. https://doi.org/10.1038/nsmb.2890.

Hoppe, T., Cassata, G., Barral, J.M., Springer, W., Hutagalung, A.H., Epstein, H.F., and Baumeister, R. (2004). Regulation of the myosin-directed chaperone UNC-45 by a novel E3/E4-multiubiquitylation complex in C. elegans. Cell 118, 337-349. https://doi. org/10.1016/j.cell.2004.07.014.

Hu, Q. D’Amora, D.R., MacNeil, L.T., Walhout, A.J.M., and Kubiseski, T.J. (2018). The Caenorhabditis elegans Oxidative Stress Response Requires the NHR-49 Transcription Factor. G3 (Bethesda). 2018 Dec 10;8(12):3857-3863. doi: 10.1534/g3.118.200727.

Huang, K.Y., Su, M.G., Kao, H.J., Hsieh, Y.C., Jhong, J.H., Cheng, K.H., Huang, H.D., and Lee, T.Y. (2016). dbPTM 2016: 10-year anniversary of a resource for post-translational modification of proteins. Nucleic Acids Res. 44, D435-46. https://doi.org/10.1093/nar/ gkv1240.

Huang, W.M., Li, Z.Y., Xu, Y.J., Wang, W., Zhou, M.G., Zhang, P., Liu, P.S., Xu, T., and Wu, Z.X. (2014). PKG and NHR-49 signalling co-ordinately regulate shortterm fasting-induced lysosomal lipid accumulation in C. elegans. Biochem. J. 461, 509-520. https://doi. org/10.1042/BJ20140191.

Irazoqui, J.E., Troemel, E.R., Feinbaum, R.L., Luhachack, L.G., Cezairliyan, B.O., and Ausubel, F.M. (2010a). Distinct pathogenesis and host responses during infection of C. elegans by P. aeruginosa and S. aureus. PLOS Pathog. 6, e1000982. https://doi.org/10.1371/ journal.ppat.1000982.

Irazoqui, J.E., Urbach, J.M., and Ausubel, F.M. (2010b). Evolution of host innate defence: insights from Caenorhabditis elegans and primitive invertebrates. Nat. Rev. Immunol. 10, 47-58. https://doi.org/10.1038/ nri2689.

Jones, D., and Candido, E.P. (1993). Novel ubiquitin-like ribosomal protein fusion genes from the nematodes Caenorhabditis elegans and Caenorhabditis briggsae. J. Biol. Chem. 268, 19545-19551.

Jones, D., Crowe, E., Stevens, T.A., and Candido, E.P. (2002). Functional and phylogenetic analysis of the ubiquitylation system in Caenorhabditis elegans: ubiquitin-conjugating enzymes, ubiquitin-activating enzymes, and ubiquitin-like proteins. Genome Biol. 3, RESEARCH0002.

Kaletta, T., and Hengartner, M.O. (2006). Finding function in novel targets: C. elegans as a model organism. Nat. Rev. Drug Discov. 5, 387-398. https://doi.org/10.1038/ $\operatorname{nrd} 2031$.

Kaminsky, R., Denison, C., Bening-Abu-Shach, U., Chisholm, A.D., Gygi, S.P., and Broday, L. (2009). SUMO regulates the assembly and function of a cytoplasmic intermediate filament protein in C. elegans.
Dev. Cell 17, 724-735. https://doi.org/10.1016/j. devcel.2009.10.005.

Keith, S.A., Maddux, S.K., Zhong, Y., Chinchankar, M.N., Ferguson, A.A., Ghazi, A., and Fisher, A.L. (2016). Graded proteasome dysfunction in Caenorhabditis elegans activates an adaptive response involving the conserved SKN-1 and ELT-2 transcription factors and the autophagy-lysosome pathway. PLOS Genet. 12, e1005823. https://doi.org/10.1371/journal. pgen. 1005823.

Kerscher, O., Felberbaum, R., and Hochstrasser, M. (2006). Modification of proteins by ubiquitin and ubiquitin-like proteins.Annu. Rev. Cell Dev.Biol.22, 159-180.https:// doi.org/10.1146/annurev.cellbio.22.010605.093503.

Khan, L.A., and Nukina, N. (2004). Molecular and functional analysis of Caenorhabditis elegans CHIP, a homologue of Mammalian CHIP. FEBS Lett. 565, 11-18. https://doi.org/10.1016/j.febslet.2004.03.084.

Kipreos, E.T. (2005). Ubiquitin-mediated pathways in C. elegans. WormBook, 1-24. https://doi.org/10.1895/ wormbook.1.36.1.

Kirkpatrick, D.S., Dale, K.V., Catania, J.M., and Gandolfi, A.J. (2003). Low-level arsenite causes accumulation of ubiquitinated proteins in rabbit renal cortical slices and HEK293 cells. Toxicol. Appl. Pharmacol. 186, 101-109.

Kulkarni, M., and Smith, H.E. (2008). E1 ubiquitinactivating enzyme UBA-1 plays multiple roles throughout $C$. elegans development. PLOS Genet. 4, e1000131. https://doi.org/10.1371/journal. pgen. 1000131.

Lewicki, M.C., Srikumar, T., Johnson, E., and Raught, B. (2015). The S. cerevisiae SUMO stress response is a conjugation-deconjugation cycle that targets the transcription machinery. J. Proteomics 118, 39-48. https://doi.org/10.1016/j.jprot.2014.11.012.

Li, W., Gao, B., Lee, S.M., Bennett, K., and Fang, D. (2007). RLE-1, an E3 ubiquitin ligase, regulates C. elegans aging by catalyzing DAF-16 polyubiquitination. Dev. Cell 12 , $235-246$.

Lim, Y., Lee, D., Kalichamy, K., Hong, S.E., Michalak, M., Ahnn, J., Kim, D.H., and Lee, S.K. (2014). Sumoylation regulates ER stress response by modulating calreticulin gene expression in XBP-1-dependent mode in Caenorhabditis elegans. Int. J. Biochem. Cell Biol. 53, 399-408. https://doi.org/10.1016/j. biocel.2014.06.005.

Livnat-Levanon, N., Kevei, É., Kleifeld, O., Krutauz, D., Segref, A., Rinaldi, T., Erpapazoglou, Z., Cohen, M., Reis, N., Hoppe, T., et al. (2014). Reversible 26S proteasome disassembly upon mitochondrial stress. Cell Rep. 7, 1371-1380.

Mattiroli, F., and Sixma, T.K. (2014). Lysine-targeting specificity in ubiquitin and ubiquitin-like modification pathways. Nat. Struct. Mol. Biol. 21, 308-316. https:// doi.org/10.1038/nsmb.2792.

Mehta, R., Steinkraus, K.A., Sutphin, G.L., Ramos, F.J., Shamieh, L.S., Huh, A., Davis, C., Chandler-Brown, D., and Kaeberlein, M. (2009). Proteasomal regulation of the hypoxic response modulates aging in C. elegans. Science 324, 1196-1198. https://doi.org/10.1126/ science. 1173507.

Miller, M.J., Scalf, M., Rytz, T.C., Hubler, S.L., Smith, L.M., and Vierstra, R.D. (2013). Quantitative proteomics 
reveals factors regulating RNA biology as dynamic targets of stress-induced SUMOylation in Arabidopsis. Mol. Cell Proteomics 12, 449-463. https://doi. org/10.1074/mcp.M112.025056.

Moll, L., Roitenberg, N., Bejerano-Sagie, M., Boocholez, H., Carvalhal Marques, F., Volovik, Y., Elami, T., Siddiqui, A.A., Grushko, D., Biram, A., et al. (2018). The Insulin/ IGF signaling cascade modulates SUMOylation to regulate aging and proteostasis in. Elife 7, pii: e38635. https://doi.org/10.7554/eLife.38635.

Oliveira, R.P., Porter Abate, J., Dilks, K., Landis, J., Ashraf, J., Murphy, C.T., and Blackwell, T.K. (2009). Condition-adapted stress and longevity gene regulation by Caenorhabditis elegans SKN-1/Nrf. Aging Cell 8, 524-541. https://doi.org/10.1111/j.14749726.2009.00501.x.

Palikaras, K., Lionaki, E., and Tavernarakis, N. (2015). Coordination of mitophagy and mitochondrial biogenesis during ageing in C. elegans. Nature 521, 525-528. https://doi.org/10.1038/nature14300.

Papaevgeniou, N., and Chondrogianni, N. (2014). The ubiquitin proteasome system in Caenorhabditis elegans and its regulation. Redox Biol. 2, 333-347. https://doi. org/10.1016/j.redox.2014.01.007.

Pelisch, F., Sonneville, R., Pourkarimi, E., Agostinho, A., Blow, J.J., Gartner, A., and Hay, R.T. (2014). Dynamic SUMO modification regulates mitotic chromosome assembly and cell cycle progression in Caenorhabditis elegans. Nat. Commun. 5, 5485. https://doi. org/10.1038/ncomms6485.

Pelisch, F., Tammsalu, T., Wang, B., Jaffray, E.G., Gartner, A., and Hay, R.T. (2017). A SUMO-dependent protein network regulates chromosome congression during oocyte meiosis. Mol. Cell 65, 66-77.

Pferdehirt, R.R., and Meyer, B.J. (2013). SUMOylation is essential for sex-specific assembly and function of the Caenorhabditis elegans dosage compensation complex on X chromosomes. Proc. Natl. Acad. Sci. U.S.A. 110, E3810-9. https://doi.org/10.1073/pnas.1315793110.

Piano, F., Schetter, A.J., Morton, D.G., Gunsalus, K.C., Reinke, V., Kim, S.K., and Kemphues, K.J. (2002). Gene clustering based on RNAi phenotypes of ovary-enriched genes in C. elegans. Curr. Biol. 12, 1959-1964.

Pinto, M.P., Carvalho, A.F., Grou, C.P., Rodríguez-Borges, J.E., Sá-Miranda, C., and Azevedo, J.E. (2012). Heat shock induces a massive but differential inactivation of SUMO-specific proteases. Biochim. Biophys. Acta 1823, 1958-1966. https://doi.org/10.1016/j. bbamcr.2012.07.010.

Princz, A., and Tavernarakis, N. (2017). The role of SUMOylation in ageing and senescent decline. Mech. Ageing Dev. 162, 85-90.

Prudden, J., Pebernard, S., Raffa, G., Slavin, D.A., Perry, J.J., Tainer, J.A., McGowan, C.H., and Boddy, M.N. (2007). SUMO-targeted ubiquitin ligases in genome stability. EMBO J. 26, 4089-4101.

Psakhye, I., and Jentsch, S. (2012). Protein group modification and synergy in the SUMO pathway as exemplified in DNA repair. Cell 151, 807-820.

Ratnappan, R., Amrit, F.R., Chen, S.W., Gill, H., Holden, K., Ward, J., Yamamoto, K.R., Olsen, C.P., and Ghazi, A. (2014). Germline signals deploy NHR-49 to modulate fatty-acid $\beta$-oxidation and desaturation in somatic tissues of C. elegans. PLOS Genet. 10, e1004829. https://doi. org/10.1371/journal.pgen.1004829.

Reddy, K.C., Dror, T., Sowa, J.N., Panek, J., Chen, K., Lim, E.S., Wang, D., and Troemel, E.R. (2017). An intracellular pathogen response pathway promotes proteostasis in C. elegans. Curr. Biol. 27, 3544-3553.e5.

Ribet, D., and Cossart, P. (2018). Ubiquitin, SUMO, and NEDD8: key targets of bacterial pathogens. Trends Cell Biol. 28, 926-940.

Rual, J.F., Ceron, J., Koreth, J., Hao, T., Nicot, A.S., Hirozane-Kishikawa, T., Vandenhaute, J., Orkin, S.H., Hill, D.E., van den Heuvel, S., et al. (2004). Toward improving Caenorhabditis elegans phenome mapping with an ORFeome-based RNAi library. Genome Res. 14, 2162-2168.

Saitoh, H., and Hinchey, J. (2000). Functional heterogeneity of small ubiquitin-related protein modifiers SUMO-1 versus SUMO-2/3. J. Biol. Chem. 275, 6252-6258.

Sapir, A., Tsur, A., Koorman, T., Ching, K., Mishra, P., Bardenheier, A., Podolsky, L., Bening-Abu-Shach, U., Boxem, M., Chou, T.F., et al. (2014). Controlled sumoylation of the mevalonate pathway enzyme HMGS-1 regulates metabolism during aging. Proc. Natl. Acad. Sci. U.S.A. 111, E3880-9. https://doi. org/10.1073/pnas.1414748111.

Sem, X., and Rhen, M. (2012). Pathogenicity of Salmonella enterica in Caenorhabditis elegans relies on disseminated oxidative stress in the infected host. PLOS ONE 7, e45417. https://doi.org/10.1371/journal. pone.0045417.

Shen, T.H., Lin, H.K., Scaglioni, P.P., Yung, T.M., and Pandolfi, P.P. (2006). The mechanisms of PML-nuclear body formation. Mol. Cell 24, 331-339.

Shimada, M., Kanematsu, K., Tanaka, K., Yokosawa, H., and Kawahara, H. (2006). Proteasomal ubiquitin receptor RPN-10 controls sex determination in Caenorhabditis elegans. Mol. Biol. Cell 17, 5356-5371.

Skaar, J.R., Pagan, J.K., and Pagano, M. (2013). Mechanisms and function of substrate recruitment by F-box proteins. Nat. Rev. Mol. Cell Biol. 14, 369-381. https://doi. org/10.1038/nrm3582.

Song, J., Durrin, L.K., Wilkinson, T.A., Krontiris, T.G., and Chen, Y. (2004). Identification of a SUMO-binding motif that recognizes SUMO-modified proteins. Proc. Natl. Acad. Sci. U.S.A. 101, 14373-14378. https://doi. org/10.1073/pnas.0403498101.

Song, J., Zhang, Z., Hu, W., and Chen, Y. (2005). Small ubiquitin-like modifier (SUMO) recognition of a SUMO binding motif: a reversal of the bound orientation. J. Biol. Chem. 280, 40122-40129.

Squiban, B., and Kurz, C.L. (2011). C. elegans: an all in one model for antimicrobial drug discovery. Curr. Drug Targets 12, 967-977.

Stanhill, A., Haynes, C.M., Zhang, Y., Min, G., Steele, M.C., Kalinina, J., Martinez, E., Pickart, C.M., Kong, X.P., and Ron, D. (2006). An arsenite-inducible 19S regulatory particle-associated protein adapts proteasomes to proteotoxicity. Mol. Cell 23, 875-885.

Stankovic-Valentin, N., Drzewicka, K., König, C., Schiebel, E., and Melchior, F. (2016). Redox regulation of SUMO enzymes is required for ATM activity and survival in oxidative stress. EMBO J. 35, 1312-1329. https://doi. org/10.15252/embj.201593404. 
Surana, P., Gowda, C.M., Tripathi, V., Broday, L., and Das, R. (2017). Structural and functional analysis of SMO-1, the SUMO homolog in Caenorhabditis elegans. PLOS ONE 12, e0186622. https://doi.org/10.1371/journal. pone. 0186622 .

Sönnichsen, B., Koski, L.B., Walsh, A., Marschall, P., Neumann, B., Brehm, M., Alleaume, A.M., Artelt, J., Bettencourt, P., Cassin, E., et al. (2005). Full-genome RNAi profiling of early embryogenesis in Caenorhabditis elegans. Nature 434, 462-469.

Tawo, R., Pokrzywa, W., Kevei, É., Akyuz, M.E., Balaji, V., Adrian, S., Höhfeld, J., and Hoppe, T. (2017). The ubiquitin ligase chip integrates proteostasis and aging by regulation of insulin receptor turnover. Cell 169, 470-482.e13.

Thomas, J.H. (2006). Adaptive evolution in two large families of ubiquitin-ligase adapters in nematodes and plants. Genome Res. 16, 1017-1030.

Tsur, A., Bening Abu-Shach, U., and Broday, L. (2015). ULP-2 SUMO protease regulates E-cadherin recruitment to adherens junctions. Dev. Cell 35, 63-77. https://doi.org/10.1016/j.devcel.2015.08.019.

Uzunova, K., Göttsche, K., Miteva, M., Weisshaar, S.R., Glanemann, C., Schnellhardt, M., Niessen, M., Scheel, H., Hofmann, K., Johnson, E.S., et al. (2007). Ubiquitindependent proteolytic control of SUMO conjugates. J. Biol. Chem. 282, 34167-34175.

Van Gilst, M.R., Hadjivassiliou, H., and Yamamoto, K.R. (2005). A Caenorhabditis elegans nutrient response system partially dependent on nuclear receptor NHR49. Proc. Natl. Acad. Sci. U.S.A. 102, 13496-13501.
Vartiainen, S., Pehkonen, P., Lakso, M., Nass, R., and Wong, G. (2006). Identification of gene expression changes in transgenic $C$. elegans overexpressing human alphasynuclein. Neurobiol. Dis. 22, 477-486.

Vilchez, D., Morantte, I., Liu, Z., Douglas, P.M., Merkwirth, C., Rodrigues, A.P., Manning, G., and Dillin, A. (2012). RPN-6 determines C. elegans longevity under proteotoxic stress conditions. Nature 489, 263-268. https://doi.org/10.1038/nature11315.

Walther, D.M., Kasturi, P., Zheng, M., Pinkert, S., Vecchi, G., Ciryam, P., Morimoto, R.I., Dobson, C.M., Vendruscolo, M., Mann, M., et al. (2015). Widespread proteome remodeling and aggregation in aging C. elegans. Cell 161, 919-932. https://doi.org/10.1016/j.cell.2015.03.032.

Wu, C.W., Wang, Y., and Choe, K.P. (2017). F-box protein XREP-4 is a new regulator of the oxidative stress response in Caenorhabditis elegans. Genetics 206, 859871. https://doi.org/10.1534/genetics.117.200592.

Yashiroda, H., Mizushima, T., Okamoto, K., Kameyama, T., Hayashi, H., Kishimoto, T., Niwa, S., Kasahara, M., Kurimoto, E., Sakata, E., et al. (2008). Crystal structure of a chaperone complex that contributes to the assembly of yeast $20 \mathrm{~S}$ proteasomes. Nat. Struct. Mol. Biol. 15, 228-236. https://doi.org/10.1038/nsmb.1386.

Zugasti, O., Thakur, N., Belougne, J., Squiban, B., Kurz, C.L., Soulé, J., Omi, S., Tichit, L., Pujol, N., and Ewbank, J.J. (2016). A quantitative genome-wide RNAi screen in C. elegans for antifungal innate immunity genes. BMC Biol. 14, 35. https://doi.org/10.1186/s12915-016-0256-3. 